\title{
Fixed point theorems for contractions in fuzzy normed spaces and intuitionistic fuzzy normed spaces
}

\author{
Jiang Zhu' ${ }^{1}$ Yunjie Wang ${ }^{2^{*}}$ and Cheng-Cheng Zhu ${ }^{3}$
}

${ }^{*}$ Correspondence:
wangyunjiemath@163.com
${ }^{2}$ Kewen Institute, Jiangsu Normal
University, Xuzhou, 221116, People's
Republic of China
Full list of author information is
available at the end of the article

\begin{abstract}
In this paper, we prove that some coupled fixed point theorems and coupled coincidence point theorems for contractions in fuzzy normed spaces and intuitionistic fuzzy normed spaces can be directly deduced from fixed point theorems for contractions in fuzzy normed spaces. We also prove that these results are equivalent.

MSC: Primary 47H10; secondary 54A40; 54E50; 54H25

Keywords: coupled fixed point; coupled coincidence; intuitionistic fuzzy normed space; partially ordered set; mixed monotone mapping
\end{abstract}

\section{Introduction}

The well-known Banach contraction mapping principle [1] is a powerful tool in nonlinear analysis; many mathematicians have much contributed to the improvement and generalization of this principle in many ways. Especially, some recent meaningful results have been obtained in [2-18].

In this paper, we first prove a simple fixed point theorem for an increasing mapping defined on fuzzy normed spaces, and by using this result, we can easily prove some coupled fixed point theorems and coupled coincidence point theorems in fuzzy normed spaces and intuitionistic fuzzy normed spaces. Also, we prove that these results are essentially equivalent. Finally, we give an example to show that our contractive conditions is a real improvement over the contractive conditions used in [13] and [17]. Our results are also an improvement over the results in [13] and [17].

For the reader's convenience, we restate some definitions and results that will be used in this paper.

Definition $1.1([13])$ A binary operation $*:[0,1] \times[0,1] \rightarrow[0,1]$ is a continuous $t$-norm if $*$ satisfies the following conditions:

(i) $*$ is commutative and associative;

(ii) $*$ is continuous;

(iii) $a * 1=a, \forall a \in[0,1]$;

(iv) $a * b \leq c * d$, whenever $a \leq c$ and $b \leq d$ for all $a, b, c, d \in[0,1]$.

(0) 2013 Zhu et al.; licensee Springer. This is an Open Access article distributed under the terms of the Creative Commons Attribution License (http://creativecommons.org/licenses/by/2.0), which permits unrestricted use, distribution, and reproduction in any medium, provided the original work is properly cited. 
Definition 1.2 ( $c f .[14,18])$ A $t$-norm $*$ is said to be of $\mathrm{H}$-type if the sequence of functions $\left\{*^{n} a\right\}_{n=1}^{\infty}$ is equicontinuous at $a=1$.

The $t$-norm $*_{m}$ defined by $a *_{m} b=\min \{a, b\}$ is an example of an H-type $t$-norm $*$.

Definition $1.3([13])$ A binary operation $\star:[0,1] \times[0,1] \rightarrow[0,1]$ is a continuous $t$-conorm if $\star$ satisfies the following conditions:

(i) $\star$ is commutative and associative;

(ii) $\star$ is continuous;

(iii) $a \star 0=a, \forall a \in[0,1]$;

(iv) $a \star b \leq c \star d$, whenever $a \leq c$ and $b \leq d$ for all $a, b, c, d \in[0,1]$.

Definition 1.4 ([23]) A fuzzy normed space (briefly, FNS) is a triple $(X, \mu, *)$, where $X$ is a vector space, $*$ is a continuous $t$-norm and $\mu: X \times(0, \infty) \rightarrow[0,1]$ is a fuzzy set such that, for all $x, y \in X$ and $t, s>0$,

(i) $\mu(x, t)>0$;

(ii) $\mu(x, t)=1$ if and only if $x=\theta$;

(iii) $\mu(c x, t)=\mu\left(x, \frac{t}{|c|}\right)$ for all $c \neq 0$;

(iv) $\mu(x, s) * \mu(y, t) \leq \mu(x+y, s+t)$;

(v) $\mu(x, \cdot)$ is a continuous function of $\mathbb{R}^{+}$and

$$
\lim _{t \rightarrow \infty} \mu(x, t)=1, \quad \lim _{t \rightarrow 0} \mu(x, t)=0 .
$$

By the results in George and Veeramani [19], we can know that every fuzzy norm $(\mu, *)$ on $X$ generates a Hausdorff first countable topology $\tau_{\mu}$ on $X$ which has as a base the family of open sets of the form

$$
\{B(x, r, t): x \in X, r \in(0,1), t>0\},
$$

where $B(x, r, t)=\{y \in X: \mu(x, y, t)>1-r\}$ for all $x \in X, r \in(0,1)$ and $t>0$.

Definition $1.5(c f .[13,21])$ The 5-tuple $(X, \mu, v, *, \star)$ is said to be an intuitionistic fuzzy normed space (for short, IFNS) if $X$ is a linear space, $*$ is a continuous $t$-norm, $\star$ is a continuous $t$-conorm and $\mu, v$ are fuzzy sets on $X \times(0, \infty)$ satisfying the following conditions:

(i) $\mu(x, t)+v(x, t) \leq 1, \forall(x, t) \in X \times(0, \infty)$;

(ii) $\mu(x, t)>0$;

(iii) $\mu(x, t)=1$ if and only if $x=\theta$;

(iv) $\mu(c x, t)=\mu\left(x, \frac{t}{|c|}\right)$ for all $c \neq 0$;

(v) $\mu(x, s) * \mu(y, t) \leq \mu(x+y, s+t)$;

(vi) $\mu(x, \cdot)$ is a continuous function of $\mathbb{R}^{+}$and

$$
\lim _{t \rightarrow \infty} \mu(x, t)=1, \quad \lim _{t \rightarrow 0} \mu(x, t)=0
$$

(vii) $v(x, t)<1$;

(viii) $v(x, t)=0$ if and only if $x=\theta$;

(ix) $v(c x, t)=v\left(x, \frac{t}{|c|}\right)$ for all $c \neq 0$; 
(x) $v(x, s) \star v(y, t) \geq v(x+y, s+t) ;$

(xi) $v(x, \cdot)$ is a continuous function of $\mathbb{R}^{+}$and

$$
\lim _{t \rightarrow \infty} v(x, t)=0, \quad \lim _{t \rightarrow 0} v(x, t)=1 .
$$

Park proved in [22], among other results, that each intuitionistic fuzzy norm $(\mu, v)$ on $X$ generates a Hausdorff first countable topology $\tau_{(\mu, v)}$ on $X$ which has as a base the family of open sets of the form $\{B(x, r, t): x \in X, r \in(0,1), t>0\}$, where $B(x, r, t)=\{y \in X: \mu(x, y, t)>$ $1-r, v(x, y, t)<r\}$ for all $x \in X, r \in(0,1)$ and $t>0$. According to this topology, Park [22] gave the following definitions.

Definition 1.6 A sequence $\left\{x_{n}\right\}$ in an intuitionistic fuzzy normed linear space $(X, \mu, \nu, *, \star)$ is said to converge to $x \in X$ with respect to the intuitionistic fuzzy norm $(\mu, \nu)$ if, for any $\varepsilon>0, t>0,0<\varepsilon<1$, there exists an integer $n_{0} \in \mathbb{N}$ such that

$$
\mu\left(x_{n}-x, t\right)>1-\varepsilon \quad \text { and } \quad v\left(x_{n}-x, t\right)<\varepsilon \quad \text { for all } n \geq n_{0} .
$$

Definition 1.7 A sequence $\left\{x_{n}\right\}$ in an intuitionistic fuzzy normed linear space $(X, \mu, v, *, \star)$ is said to be a Cauchy sequence with respect to the intuitionistic fuzzy norm $(\mu, v)$ if, for any $\varepsilon>0, t>0,0<\varepsilon<1$, there exists an integer $n_{0} \in \mathbb{N}$ such that

$$
\mu\left(x_{m}-x_{n}, t\right)>1-\varepsilon \quad \text { and } \quad \nu\left(x_{m}-x_{n}, t\right)<\varepsilon \quad \text { for all } m, n \geq n_{0} .
$$

Definition 1.8 Let $(X, \mu, v, *, \star)$ be an IFNS. Then $(X, \mu, v, *, \star)$ is said to be complete if every Cauchy sequence in $(X, \mu, v, *, \star)$ is convergent.

Definition 1.9 Let $X$ and $Y$ be two intuitionistic fuzzy normed spaces. A mapping $f$ : $X \rightarrow Y$ is said to be continuous at $x_{0} \in X$ if, for any sequence $\left\{x_{n}\right\}$ in $X$ converging to $x_{0}$, the sequence $\left\{f\left(x_{n}\right)\right\}$ in $Y$ converges to $f\left(x_{0}\right) \in Y$. If $f: X \rightarrow Y$ is continuous at each $x \in X$, then $f$ is said to be continuous on $X$.

For the topology $\tau_{(\mu, v)}$, Gregori et al. [20] proved the following result.

Lemma 1.1 Let $(X, \mu, v, *, \star)$ be an intuitionistic fuzzy metric space. Then the topologies $\tau_{(\mu, \nu)}$ and $\tau_{\mu}$ coincide on $X$.

The following lemma was proved by Haghi et al. [15].

Lemma 1.2 Let $X$ be a nonempty set, and let $g: X \rightarrow X$ be a mapping. Then there exists a subset $E \subset X$ such that $g(E)=g(X)$ and $g: E \rightarrow X$ is one-to-one.

Definition 1.10 ([16]) A point $(x, y) \in X \times X$ is called a coupled coincidence point of the mappings $F: X \times X \rightarrow X$ and $g: X \rightarrow X$ if

$$
F(x, y)=g x, \quad F(y, x)=g y .
$$


Definition 1.11 ([16]) Let $(X, \sqsubseteq$ ) be a partially ordered set, and let $F: X \times X \rightarrow X, g$ : $X \rightarrow X$ be two mappings. Then $F$ is said to have the mixed $g$-monotone property if $F$ is monotone $g$-non-decreasing in the first argument and is monotone $g$-non-increasing in the second argument, that is, for any $x, y \in X$,

$$
x_{1}, x_{2} \in X, \quad g\left(x_{1}\right) \sqsubseteq g\left(x_{2}\right) \quad \Longrightarrow \quad F\left(x_{1}, y\right) \sqsubseteq F\left(x_{2}, y\right),
$$

and

$$
y_{1}, y_{2} \in X, \quad g\left(y_{1}\right) \sqsubseteq g\left(y_{2}\right) \quad \Longrightarrow \quad F\left(x, y_{1}\right) \sqsupseteq F\left(x, y_{2}\right) .
$$

If $g: X \rightarrow X$ is an identity mapping, we say that $F$ has the mixed monotone property.

\section{Main results}

Theorem 2.1 Let $(X, \sqsubseteq)$ be a partially ordered set, and let $(X, \mu, *)$ be a complete FNS such that the t-norm $*$ is of H-type. Let $F: X \rightarrow X$ be a mapping such that $F$ is non-decreasing and

$$
\mu(F(x)-F(u), k t) \geq *^{2} \mu(x-u, t)
$$

for which $x \sqsubseteq u$ and $t>0$, where $0<k<1$. Suppose either

(a) $F$ is continuous, or

(b) if $\left\{x_{n}\right\}$ is a non-decreasing sequence and $\lim _{n \rightarrow \infty} x_{n}=x$, then $x_{n} \sqsubseteq x$ for all $n \in \mathbb{N}$.

If there exists $x_{0} \in X$ such that

$$
x_{0} \sqsubseteq F\left(x_{0}\right),
$$

then $F$ has a fixed point in $X$.

Proof Let $x_{0} \in X$ such that $x_{0} \sqsubseteq F\left(x_{0}\right)$, and let $x_{n}=F\left(x_{n-1}\right), n=1,2, \ldots$, then we have that

$$
x_{0} \sqsubseteq x_{1} \sqsubseteq x_{2} \sqsubseteq \cdots \sqsubseteq x_{n} \sqsubseteq x_{n+1} \sqsubseteq \cdots .
$$

Now, put

$$
\delta_{n}(t):=\mu\left(x_{n}-x_{n+1}, t\right) .
$$

Then, by using (2.1), we have

$$
\mu\left(x_{n}-x_{n+1}, k t\right)=\mu\left(F\left(x_{n-1}\right)-F\left(x_{n}\right), k t\right) \geq *^{2} \mu\left(x_{n-1}-x_{n}, t\right)=*^{2} \delta_{n-1}(t) .
$$

Thus, it follows that $\delta_{n}(k t) \geq *^{2} \delta_{n-1}(t)$, and so

$$
\delta_{n}(t) \geq *^{2} \delta_{n-1}\left(\frac{t}{k}\right) \geq *^{2^{n}} \delta_{0}\left(\frac{t}{k^{n}}\right)
$$


On the other hand, we have

$$
t(1-k)\left(1+k+\cdots+k^{m-n-1}\right)<t, \quad \forall m>n, 0<k<1 .
$$

By Definition 1.4, we get that

$$
\begin{aligned}
\mu\left(x_{n}-x_{m}, t\right) \geq & \mu\left(x_{n}-x_{m}, t(1-k)\left(1+k+\cdots+k^{m-n-1}\right)\right) \\
\geq & \mu\left(x_{n}-x_{n+1}, t(1-k)\right) * \mu\left(x_{n+1}-x_{m}, t(1-k)\left(k+\cdots+k^{m-n-1}\right)\right) \\
\geq & \mu\left(x_{n}-x_{n+1}, t(1-k)\right) * \mu\left(x_{n+1}-x_{n+2}, t(1-k) k\right) \\
& * \cdots * \mu\left(x_{m-1}-x_{m}, t(1-k) k^{m-n-1}\right) .
\end{aligned}
$$

It follows from (2.2) and (2.3) that

$$
\begin{aligned}
\mu\left(x_{n}-x_{m}, t\right) \geq & \mu\left(x_{n}-x_{n+1}, t(1-k)\right) * \mu\left(x_{n+1}-x_{n+2}, t(1-k) k\right) \\
& * \cdots * \mu\left(x_{m-1}-x_{m}, t(1-k) k^{m-n-1}\right) \\
\geq & {\left[*^{2^{n}} \delta_{0}\left(\frac{t(1-k)}{k^{n}}\right)\right] * \cdots *\left[*^{2^{m-1}} \delta_{0}\left(\frac{t(1-k)}{k^{n}}\right)\right] } \\
= & *^{2^{m}-2^{n}} \delta_{0}\left(\frac{t(1-k)}{k^{n}}\right) .
\end{aligned}
$$

By the hypothesis, the $t$-norm $*$ is of $\mathrm{H}$-type; for all $\varepsilon \in(0,1)$, there exists $\eta>0$ such that

$$
*^{p}(s)>1-\varepsilon,
$$

for all $s \in(1-\eta, 1]$ and for all $p$. Note that

$$
\lim _{n \rightarrow \infty} \delta_{0}\left(\frac{t(1-k)}{k^{n}}\right)=1
$$

for all $t>0$ and $0<k<1$, we have that there exists $n_{0}$ such that

$$
\mu\left(x_{n}-x_{m}, t\right)>1-\varepsilon,
$$

for all $m>n>n_{0}$. Thus, $\left\{x_{n}\right\}$ is a Cauchy sequence. Since $X$ is complete, there exists $x \in X$ such that

$$
\lim _{n \rightarrow \infty} x_{n}=x .
$$

If the assumption (a) holds, then by the continuity of $F$, we get that

$$
x=\lim _{n \rightarrow \infty} x_{n+1}=\lim _{n \rightarrow \infty} F\left(x_{n}\right)=F(x) .
$$

If the assumption (b) holds, then we have that $x_{n} \sqsubseteq x$ for all $n \in \mathbb{N}$. It follows from (2.1) that

$$
\lim _{n \rightarrow \infty} \mu\left(x_{n+1}-F(x), k t\right)=\lim _{n \rightarrow \infty} \mu\left(F\left(x_{n}\right)-F(x), k t\right) \geq \lim _{n \rightarrow \infty} *^{2} \mu\left(x_{n}-x, t\right)=1 .
$$


Thus, $\mu(x-F(x), k t)=1$, that is, $x=F(x)$. Therefore, $x$ is a fixed point of $F$. The proof is completed.

Theorem 2.2 Let $(X$, $)$ be a partially ordered set, and let $(X, \mu, *)$ be a complete FNS such that the $t$-norm $*$ is of H-type and $a * b>0$ for any $a, b \in(0,1]$. Let $F: X \times X \rightarrow X$ be $a$ mapping such that $F$ has the mixed monotone property and

$$
\mu(F(x, y)-F(u, v), k t) \geq \mu(x-u, t) * \mu(y-v, t)
$$

for which $x \sqsubseteq u, y \sqsupseteq v$ and $t>0$, where $0<k<1$. Suppose either

(a) $F$ is continuous, or

(b) $X$ has the following property:

(i) if $\left\{x_{n}\right\}$ is a non-decreasing sequence and $\lim _{n \rightarrow \infty} x_{n}=x$, then $x_{n} \sqsubseteq x$ for all $n \in \mathbb{N}$,

(ii) if $\left\{y_{n}\right\}$ is a non-increasing sequence and $\lim _{n \rightarrow \infty} y_{n}=y$, then $y_{n} \sqsupseteq y$ for all $n \in \mathbb{N}$.

If there exist $x_{0}, y_{0} \in X$ such that

$$
x_{0} \sqsubseteq F\left(x_{0}, y_{0}\right), \quad y_{0} \sqsupseteq F\left(y_{0}, x_{0}\right),
$$

then $F$ has a coupled fixed point $x, y \in X$, that is,

$$
x=F(x, y), \quad y=F(y, x) .
$$

Proof First, we define a partial order $\preccurlyeq$ on $X \times X$ as follows: $(x, y) \preccurlyeq(u, v)$ if and only if $x \sqsubseteq u$ and $y \sqsupseteq v$. Second, we define a fuzzy set on $X \times X$ as follows: $\tilde{\mu}((x, y), t)=\mu(x, t) * \mu(y, t)$ for any $(x, y) \in X \times X$ and any $t>0$. Since $(X, \mu, *)$ is a complete FNS, we can easily prove that $(X \times X, \tilde{\mu}, *)$ is a complete FNS. Lastly, we define a mapping $\widetilde{F}: X \times X \rightarrow X \times X$ by

$$
\widetilde{F}(x, y)=(F(x, y), F(y, x)), \quad \forall(x, y) \in X \times X
$$

Since $F$ has the mixed monotone property, if $(x, y) \preccurlyeq(u, v)$, we have that

$$
\begin{aligned}
& F(x, y) \sqsubseteq F(u, y) \sqsubseteq F(u, v), \\
& F(v, u) \sqsubseteq F(y, u) \sqsubseteq F(y, x),
\end{aligned}
$$

that is, $\widetilde{F}(x, y) \preccurlyeq \widetilde{F}(u, v)$. Therefore, $\widetilde{F}: X \times X \rightarrow X \times X$ is a non-decreasing mapping. Since $x_{0}, y_{0} \in X$ and

$$
x_{0} \sqsubseteq F\left(x_{0}, y_{0}\right), \quad y_{0} \sqsupseteq F\left(y_{0}, x_{0}\right),
$$

we have that $\left(x_{0}, y_{0}\right) \preccurlyeq\left(F\left(x_{0}, y_{0}\right), F\left(y_{0}, x_{0}\right)\right)=\widetilde{F}\left(x_{0}, y_{0}\right)$. If $(x, y) \preccurlyeq(u, v)$, by $(2.5)$ we have that

$$
\begin{aligned}
\tilde{\mu}(\widetilde{F}(x, y)-\widetilde{F}(u, v), k t) & =\widetilde{\mu}((F(x, y)-F(u, v), F(v, u)-F(y, x)), k t) \\
& =\mu(F(x, y)-F(u, v), k t) * \mu(F(v, u)-F(y, x), k t) \\
& \geq(\mu(x-u, t) * \mu(y-v, t)) *(\mu(x-u, t) * \mu(y-v, t)) \\
& =*^{2} \tilde{\mu}((x, y)-(u, v), t) .
\end{aligned}
$$


Thus, all the assumptions of Theorem 2.1 hold for $\widetilde{F}$ and $(X \times X, \tilde{\mu}, *)$. By Theorem 2.1 we get that $\widetilde{F}$ has a fixed point $(x, y) \in X \times X$, that is, $(x, y)=(F(x, y), F(y, x))$. This implies that $x=F(x, y), y=F(y, x)$, that is, $(x, y)$ is a coupled fixed point of $F$. The proof is completed.

By using Theorem 2.2, we can prove the following coupled fixed point theorem in intuitionistic fuzzy normed spaces.

Theorem 2.3 Let $(X, \sqsubseteq)$ be a partially ordered set, and let $(X, \mu, \nu, *, \star)$ be a complete IFNS such that the $t$-norm $*$ is of H-type and $a * b>0$ for any $a, b \in(0,1]$. Let $F: X \times X \rightarrow X$ be a mapping such that $F$ has the mixed monotone property and

$$
\mu(F(x, y)-F(u, v), k t) \geq \mu(x-u, t) * \mu(y-v, t)
$$

for which $x \sqsubseteq u, y \sqsupseteq v$ and $t>0$, where $0<k<1$. Suppose either

(a) $F$ is continuous, or

(b) $X$ has the following property:

(i) if $\left\{x_{n}\right\}$ is a non-decreasing sequence and $\lim _{n \rightarrow \infty} x_{n}=x$, then $x_{n} \sqsubseteq x$ for all $n \in \mathbb{N}$,

(ii) if $\left\{y_{n}\right\}$ is a non-increasing sequence and $\lim _{n \rightarrow \infty} y_{n}=y$, then $y_{n} \sqsupseteq y$ for all $n \in \mathbb{N}$.

If there exist $x_{0}, y_{0} \in X$ such that

$$
x_{0} \sqsubseteq F\left(x_{0}, y_{0}\right), \quad y_{0} \sqsupseteq F\left(y_{0}, x_{0}\right),
$$

then $F$ has a coupled fixed point $x, y \in X$, that is,

$$
x=F(x, y), \quad y=F(y, x) .
$$

Proof Assume that $\left\{x_{n}\right\}$ is a sequence in $(X, \mu, v, *, \star)$. Let $t>0,0<\varepsilon<1$. If $\mu\left(x_{m}-x_{n}, t\right)>$ $1-\varepsilon$, then by Definition 1.5(i) we can deduce that $v\left(x_{m}-x_{n}, t\right)<\varepsilon$. Thus, a sequence $\left\{x_{n}\right\}$ in $(X, \mu, \nu, *, \star)$ is Cauchy if and only if $\left\{x_{n}\right\}$ is Cauchy in $(X, \mu, *)$. By Lemma 1.1, we know that the topology of $(X, \mu, \nu, *, \star)$ is the same as the topology of $(X, \mu, *)$. This implies that $(X, \mu, \nu, *, \star)$ is a complete IFNS if and only if $(X, \mu, *)$ is a complete FNS. Therefore, by using Theorem 2.2 to $(X, \mu, *)$ and $F$, we get that $F$ has a coupled fixed point $x, y \in X$. The proof is completed.

Theorem 2.4 Let $(X, \sqsubseteq)$ be a partially ordered set, and let $(X, \mu, \nu, *, \star)$ be a complete IFNS such that the $t$-norm $*$ is of H-type and $a * b>0$ for any $a, b \in(0,1]$. Let $F: X \times X \rightarrow X$, $g: X \rightarrow X$ be two mappings such that $F$ has the mixed $g$-monotone property and

$$
\mu(F(x, y)-F(u, v), k t) \geq \mu(g x-g u, t) * \mu(g y-g v, t),
$$

for which $g(x) \sqsubseteq g(u), g(y) \sqsupseteq g(v)$ and $t>0$, where $0<k<1, F(X \times X) \subseteq g(X)$ and $g$ is continuous. Suppose either

(a) $F$ is continuous, or

(b) $X$ has the following property:

(i) if $\left\{x_{n}\right\}$ is a non-decreasing sequence and $\lim _{n \rightarrow \infty} x_{n}=x$, then $g x_{n} \sqsubseteq g x$ for all $n \in \mathbb{N}$, 
(ii) if $\left\{y_{n}\right\}$ is a non-increasing sequence and $\lim _{n \rightarrow \infty} y_{n}=y$, then gy $y_{n} \sqsupseteq$ gy for all $n \in \mathbb{N}$.

If there exist $x_{0}, y_{0} \in X$ such that

$$
g\left(x_{0}\right) \sqsubseteq F\left(x_{0}, y_{0}\right), \quad g\left(y_{0}\right) \sqsupseteq F\left(y_{0}, x_{0}\right),
$$

then there exist $x, y \in X$ such that

$$
g(x)=F(x, y), \quad g(y)=F(y, x)
$$

that is, $F$ and $g$ have a coupled coincidence point in $X$.

Proof The conclusion of Theorem 2.4 can be proved by using Lemma 1.2 and Theorem 2.3. Since the proof is similar to the proof of Theorem 3.2 in [17], we delete the details of the proof. The proof is completed.

Remark 2.1 It follows from the proof of the above theorems that the following implications hold: Theorem $2.1 \Longrightarrow$ Theorem $2.2 \Longrightarrow$ Theorem $2.3 \Longrightarrow$ Theorem 2.4 . Conversely, it is clear that the following implications hold: Theorem $2.4 \Longrightarrow$ Theorem $2.3 \Longrightarrow$ Theorem 2.2. Thus, we have the following conclusion.

\section{Theorem 2.5 Theorem 2.2-Theorem 2.4 are equivalent.}

Remark 2.2 In [13] and [17], the condition $a \star b \leq a b$ for all $a, b \in[0,1]$ is used. But this condition cannot hold in intuitionistic fuzzy normed spaces. In fact, if this condition holds, by using (iii) and (iv) in the definition of IFNS, we can get $1=1 \star 0 \leq 1 \cdot 0=0$, which yields a contradiction. Furthermore, the proofs of the results in [13] and [17] have the same errors as noted in [18]. Therefore, our results improve and correct results in [13] and [17].

In the following, we give an example to show that our contractive conditions are a real improvement over the contractive conditions used in [13] and [17].

Example 2.1 Let $X=\mathbb{R}, \mu(x, t)=\frac{t}{t+|x|}, v(x, t)=1-\frac{t}{t+|x|}$ for every $x \in X$, and let $t>0$, $a * b=\min \{a, b\}, a \star b=\max \{a, b\}$ for all $a, b \in[0,1]$. Then $(X, \mu, v, *, \star)$ is a complete intuitionistic fuzzy normed linear space, and the $t$-norm $*$ and $t$-conorm $\star$ are of $\mathrm{H}$-type. If $X$ is endowed with the usual order $x \sqsubseteq y \Leftrightarrow x-y \leq 0$, then $(X, \sqsubseteq)$ is a partially ordered set. Let $0<k<1$, and define $F(x, y)=\frac{x-y}{4}, g x=\frac{x}{k}$ for any $x, y \in X$. Then $F: X \times X \rightarrow X$ is a mixed $g$-monotone mapping, $F(X, X) \subseteq g(X)$, and $g$ is continuous. Let $x_{0}=-1$ and $y_{0}=1$, then

$$
\frac{-1}{k}=g x_{0} \sqsubseteq F\left(x_{0}, y_{0}\right)=\frac{-1}{2}, \quad \frac{1}{2}=F\left(y_{0}, x_{0}\right) \sqsubseteq \frac{1}{k}=g y_{0} .
$$

For any $x, y, u, v \in X$, with $g x \sqsubseteq g u, g v \sqsubseteq g y$, we have

$$
\begin{aligned}
\mu(F(x, y)-F(u, v), k t) & =\frac{k t}{k t+\frac{|u-x+y-v|}{4}} \\
& \geq \min \left\{\frac{k t}{k t+|u-x|}, \frac{k t}{k t+|y-v|}\right\}
\end{aligned}
$$




$$
\begin{aligned}
& =\min \left\{\frac{t}{t+|u-x| / k}, \frac{t}{t+|y-v| / k}\right\} \\
& =\min \{\mu(g x-g u, t), \mu(g y-g v, t)\} \\
& =\mu(g x-g u, t) * \mu(g y-g v, t) .
\end{aligned}
$$

Thus, all the conditions of Theorem 2.4 are satisfied. By Theorem 2.4, there is $\left(x^{*}, y^{*}\right) \in$ $X \times X$ such that $F\left(x^{*}, y^{*}\right)=g\left(x^{*}\right)$ and $F\left(y^{*}, x^{*}\right)=g\left(y^{*}\right)$. But $v$ does not satisfy the contractive conditions in [13] and [17]. In fact, for $u=x+1, y=v+1$,

$$
\begin{aligned}
v(F(x, y)-F(u, v), k t) & =1-\frac{k t}{k t+\frac{|u-x+y-v|}{4}}=1-\frac{k t}{k t+\frac{1}{2}} \\
& >\max \left\{1-\frac{k t}{k t+|u-x|}, 1-\frac{k t}{k t+|y-v|}\right\} \\
& =\max \left\{1-\frac{t}{t+|u-x| / k}, 1-\frac{t}{t+|y-v| / k}\right\} \\
& =\max \{v(g x-g u, t), v(g y-g v, t)\} \\
& =v(g x-g u, t) \star v(g y-g v, t) .
\end{aligned}
$$

This shows that $v$ does not satisfy the contractive conditions in [13] and [17].

\section{Competing interests}

The authors declare that they have no competing interests.

\section{Authors' contributions}

All authors contributed equally and significantly in writing this paper. All authors read and approved the final manuscript.

\section{Author details}

${ }^{1}$ School of Mathematical and Statistics, Jiangsu Normal University, Xuzhou, 221116, People's Republic of China. ${ }^{2}$ Kewen Institute, Jiangsu Normal University, Xuzhou, 221116, People's Republic of China. ${ }^{3}$ Department of Applied Mathematics, Lanzhou University of Technology, Lanzhou, 730050, People's Republic of China.

\section{Acknowledgements}

First, the authors are very grateful to the referees for their careful reading of the manuscript, valuable comments and suggestions. Next, this research was supported by the National Natural Science Foundation of China (11171286).

Received: 12 November 2012 Accepted: 12 March 2013 Published: 2 April 2013

\section{References}

1. Banach, S: Sur les opérations dans les ensembles abstraits et leurs applications aux éuations intérales. Fundam. Math. 3, 133-181 (1922)

2. Ćirić, LB, Agarwal, R, Samet, B: Mixed monotone generalized contractions in partially ordered probabilistic metric spaces. Fixed Point Theory Appl. 2011, 56 (2011)

3. Gordji, ME, Savadkouhi, MB: Stability of a mixed type additive, quadratic and cubic functional equation in random normed spaces. Filomat 25(3), 43-54 (2012)

4. Ćirić, LB, Abbas, M, Damjanovic, B, Saadati, R: Common fuzzy fixed point theorems in ordered metric spaces. Math. Comput. Model. 53, 1737-1741 (2011)

5. Shakeri, S, Ćirić, LB, Saadati, R: Common fixed point theorem in partially ordered L-fuzzy metric spaces. Fixed Point Theory Appl. 2010, Article ID 125082 (2010)

6. Ćirić, LB: Solving Banach fixed point principle for nonlinear contractions in probabilistic metric spaces. Nonlinear Anal. 72, 2009-2018 (2010)

7. Ćirić, LB, Mihet, D, Saadati, R: Monotone generalized contractions in partially ordered probabilistic metric spaces. Topol. Appl. 156, 2838-2844 (2009)

8. Ćirić, LB, Lakshmikantham, V: Coupled random fixed point theorems for nonlinear contractions in partially ordered metric spaces. Stoch. Anal. Appl. 27(6), 1246-1259 (2009)

9. Shakeri, S, Jalili, M, Saadati, R, Vaezpour, SM, Ćirić, LB: Quicksort algorithms: application of fixed point theorem in probabilistic quasi-metric spaces at domain of words. J. Appl. Sci. 9, 397-400 (2009)

10. Ćirić, LB: Some new results for Banach contractions and Edelstein contractive mappings on fuzzy metric spaces. Chaos Solitons Fractals 42, 146-154 (2009) 
11. Ćirić, LB, Jesic, SN, Ume, JS: The existence theorems for fixed and periodic points of nonexpansive mappings in intuitionistic fuzzy metric spaces. Chaos Solitons Fractals 37, 781-791 (2008)

12. Abbas, M, Babu, GVR, Alemayehu, GN: On common fixed points of weakly compatible mappings satisfying generalized condition. Filomat 25(2), 9-19 (2011)

13. Gordji, ME, Baghani, H, Cho, YJ: Coupled fixed point theorem for contractions in intuitionistic fuzzy normed spaces. Math. Comput. Model. 54, 1897-1906 (2011)

14. Hadžić, O, Pap, E: Fixed Point Theory in Probabilistic Metric Spaces. Kluwer Academic, Dordrecht (2001)

15. Haghi, RH, Rezapour, SH, Shahzad, N: Some fixed point generalizations are not real generalizations. Nonlinear Anal. 74, 1799-1803 (2011)

16. Lakshmikantham, V, Ćirić, LB: Coupled fixed point theorems for nonlinear contractions in partially ordered metric spaces. Nonlinear Anal. 70, 4341-4349 (2009)

17. Sintunavarat, W, Cho, YJ, Kumam, P: Coupled coincidence point theorems for contractions without commutative condition in intuitionistic fuzzy normed spaces. Fixed Point Theory Appl. 2011, 81 (2011)

18. Zhu, XH, Xiao, JZ: Note on 'Coupled fixed point theorems for contractions in fuzzy metric spaces'. Nonlinear Anal. 74, 5475-5479 (2011)

19. George, A, Veeramani, P: On some results in fuzzy metric spaces. Fuzzy Sets Syst. 64, 395-399 (1994)

20. Gregori, V, Romaguera, S, Veeramani, P: A note on intuitionstic fuzzy spaces. Chaos Solitons Fractals 28, 902-905 (2006)

21. Mursaleen, M, Mohiuddine, SA: On stability of a cubic functional equation in intuitionistic fuzzy normed spaces. Chaos Solitons Fractals 42, 2997-3005 (2009)

22. Park, JH: Intuitionistic fuzzy metric spaces. Chaos Solitons Fractals 22, 1039-1046 (2004)

23. Saadati, R, Vaezpour, S: Some results on fuzzy Banach spaces. J. Appl. Math. Comput. 17, 475-484 (2005)

doi:10.1186/1687-1812-2013-79

Cite this article as: Zhu et al.: Fixed point theorems for contractions in fuzzy normed spaces and intuitionistic fuzzy normed spaces. Fixed Point Theory and Applications 2013 2013:79.

\section{Submit your manuscript to a SpringerOpen ${ }^{\ominus}$ journal and benefit from:}

- Convenient online submission

- Rigorous peer review

- Immediate publication on acceptance

- Open access: articles freely available online

- High visibility within the field

- Retaining the copyright to your article 